\title{
Maternal Care and Hippocampal Plasticity: Evidence for Experience-Dependent Structural Plasticity, Altered Synaptic Functioning, and Differential Responsiveness to Glucocorticoids and Stress
}

\author{
Danielle L. Champagne, ${ }^{1,3}$ Rosemary C. Bagot,${ }^{4}$ Felisa van Hasselt, ${ }^{1}$ Ger Ramakers, ${ }^{2}$ Michael J. Meaney, ${ }^{4}$ \\ E. Ronald de Kloet, ${ }^{3}$ Marian Joëls, ${ }^{1}$ and Harm Krugers ${ }^{1}$ \\ ${ }^{1}$ Swammerdam Institute for Life Sciences Center for Neuroscience and ${ }^{2}$ Netherlands Institute for Neurosciences, University of Amsterdam, 1098 SM \\ Amsterdam, The Netherlands, ${ }^{3}$ Leiden/Amsterdam Center for Drug Research, Leiden University Medical Centre, 2300 RA Leiden, The Netherlands, and \\ ${ }^{4}$ Douglas Mental Health University Institute, McGill University, Montreal, Quebec, Canada H4H 1R3
}

\begin{abstract}
Maternal licking and grooming (LG) in infancy influences stress responsiveness and cognitive performance in the offspring. We examined the effects of variation in the frequency of pup LG on morphological, electrophysiological, and behavioral aspects of hippocampal synaptic plasticity under basal and stress-like conditions. We found shorter dendritic branch length and lower spine density in CA1 cells from the adult offspring of low compared with high LG offspring. We also observed dramatic effects on long-term potentiation (LTP) depending on corticosterone treatment. Low LG offspring, in contrast to those of high LG mothers, displayed significantly impaired LTP under basal conditions but surprisingly a significantly enhanced LTP in response to high corticosterone in vitro. This enhanced plasticity under conditions that mimic those of a stressful event was apparent in vivo. Adult low LG offspring displayed enhanced memory relative to high LG offspring when tested in a hippocampal-dependent, contextual fear-conditioning paradigm. Hippocampal levels of glucocorticoid and mineralocorticoid receptors were reduced in low compared with high LG offspring. Such effects, as well as the differences in dendritic morphology, likely contribute to LTP differences under resting conditions, as well as to the maternal effects on synaptic plasticity and behavior in response to elevated corticosterone levels. These results suggest that maternal effects may modulate optimal cognitive functioning in environments varying in demand in later life, with offspring of high and low LG mothers showing enhanced learning under contexts of low and high stress, respectively.
\end{abstract}

Key words: CA1 hippocampus; corticosterone; long-term potentiation; maternal behavior; morphology; synaptic plasticity

\section{Introduction}

The quality of parent-child interactions predicts the risk for psychopathology over the lifespan (Oakley Browne et al., 1995; Kendler et al., 2002; McEwen, 2003; Nemeroff, 2004a,b; Coldwell et al., 2006). Studies in the rat suggest direct effects of maternal care on the development of neural systems that regulate cognitive, emotional, and neuroendocrine responses to stress (Meaney, 2001) and thus influence vulnerability for mood disorders. In the rat, naturally occurring variations in the frequency of pup licking and grooming (LG) provided by the dam during the first week of

Received 0ct. 10, 2007; revised April 7, 2008; accepted April 24, 2008.

This work was supported by the Marie Curie Foundation (D.L.C.), the National Sciences and Engineering Research Council of Canada (D.L.C., M.J.M.), Top Institute Pharma Grant T5-209, the Royal Netherlands Academy of Arts and Sciences, and Human Frontier Science Program Grant RGP 39/2006 (M.J.M., E.R.d.K., M.J.). We thank Yanina Revsin for assistance with corticosterone radioimmunoassay as well as Joop van Heerikhuize and David Baraznji Sassoon for technical assistance with processing and image analysis of Golgi-stained tissue.

Correspondence should be addressed to Danielle L. Champagne, Department of Medical Pharmacology, Leiden/ Amsterdam Center for Drug Research, Leiden University Medical Centre, Gorlaeus Laboratoria, Einsteinweg 55, P.0. Box 9502, 2300 RA Leiden, The Netherlands. E-mail: d.champagne@chem.leidenuniv.nl.

DOI:10.1523/JNEUROSCI.0526-08.2008

Copyright $\odot 2008$ Society for Neuroscience $\quad$ 0270-6474/08/286037-09\$15.00/0 life are associated with individual differences in stress responsiveness, emotionality, and cognitive functioning in adult offspring. As adults, offspring of low compared with high LG mothers show increased hypothalamus-pituitary-adrenal (HPA) responses to stress (Liu et al., 1997; Weaver et al., 2004, 2005), enhanced emotionality (Caldji et al., 1998; Menard and Hakvoort, 2007), and impaired performance in tests of spatial learning and object recognition (Liu et al., 2000; Bredy et al., 2003, 2004; Toki et al., 2007). These effects are essentially reversed with cross fostering, suggesting a direct effect of maternal care (Francis et al., 1999; Caldji et al., 2003).

The maternal effects on behavioral and HPA responses to stress depend on, at least in part, epigenetic programming of gene expression (Meaney and Szyf, 2005; Diorio and Meaney, 2007). However, such effects, and especially those on hippocampaldependent forms of learning and memory, may also involve differences in synaptic plasticity. Indeed, maternal care alters the expression of synaptophysin, growth factors, and selected glutamate receptor subunits (Liu et al., 2000; Bredy et al., 2003, 2004; Toki et al., 2007). However, potential effects on the structure and function of hippocampal neurons have not been examined and 
Table 1. Frequency of maternal behaviors

\begin{tabular}{lcc}
\hline Maternal behaviors & Low LG $(n=10)$ & High LG $(n=13)$ \\
\hline Licking and grooming & $5.5 \pm 0.45$ & $12.95 \pm 0.54^{* *}$ \\
Arched-back nursing & $35.3 \pm 2.38$ & $40.6 \pm 2.44$ \\
Flat-back nursing & $37.2 \pm 2.86$ & $27.2 \pm 2.27^{* *}$ \\
Passive nursing & $6.7 \pm 0.83$ & $7.2 \pm 0.73$ \\
No maternal contact & $19.5 \pm 2.71$ & $19.1 \pm 0.54$
\end{tabular}

Mean ( \pm SEM) percentage of maternal behavior averaged over the first $6 \mathrm{~d}$ postpartum. The frequency of the different maternal behaviors, including $L G$, was summed over the $6 \mathrm{~d}$ period, and percentages for each maternal behavior were calculated. Dams used in the current study differed in the percentage of $L G$ and flat-back nursing averaged over the first week of life. ${ }^{* *} p<0.01$.

are of particular relevance in light of studies suggesting parental effects on hippocampal volume in humans (Vythilingam et al., 2002; Buss et al., 2007) and of consistent reports of decreased hippocampal volume in patients with mood disorders (Sheline et al., 1996; Bremner et al., 2000).

In the studies reported here, we examined the effects of variation in pup LG on the morphology of CA1 hippocampal neurons and on synaptic plasticity within this region in response to corticosteroid treatments that mimic basal versus stressful conditions (i.e., examining hippocampal function under a range of environmental conditions). Specifically, we investigated effects of maternal care on the following: (1) the morphology of CA1 neurons under resting condition, (2) LTP under low and high corticosterone (CORT) conditions (in vitro), (3) behavioral (learning/memory) performance in a fear conditioning task (high stress, in vivo), and (4) levels of hippocampal mineralocorticoid receptor (MR) and glucocorticoid receptor (GR) expression.

\section{Materials and Methods}

Maternal care characterization. Long-Evans rats were bred in our colony at Leiden University (Leiden, The Netherlands). Maternal care characterization was performed in accordance with the method described by Champagne et al. (2003). A total of five maternal observation periods per day (7:00 A.M., 10:00 A.M., 1:00 P.M., 5:00 P.M., and 9:00 P.M.) were conducted from postnatal day 1 (P1) (with day of birth being P0) until P6 (see Fig. $1 \mathrm{~A}$ ). Each observation period lasted $60 \mathrm{~min}$ and contained 21 observations, which were spaced by a 3 min interval. At the end of the $6 \mathrm{~d}$ observation period, each mother had a total of 630 observations. Among the different maternal behaviors, LG (with or without nursing), archedback nursing (dam displays an obvious arch in her back while nursing), flat-back nursing (dam engages in nursing postures with no obvious arch in her back), passive nursing (dam is lying on her side or back while nursing her pups), and no maternal contact was scored in detail.

The percentages of maternal LG and other maternal behaviors were determined over each day (see Fig. $1 B$ ) of the $6 \mathrm{~d}$ period (Table 1). Dams were sorted into low and high LG mothers according to the following criterion: one SD below (low LG) and one SD above (high LG) the cohort mean. From weaning (P21), male offspring of low and high LG mothers were group housed (four per cage) with their respective littermates, with access to food and water ad libitum. Low and high LG offspring used in the current study were between 2 and 3 months of age. Animals were kept at $20-22^{\circ} \mathrm{C}, 55 \% \pm 15$ humidity, on a $12 \mathrm{~h}$ light/dark cycle (lights on at 8:00 A.M.). All experimental procedures were approved by the local committee on animal bioethics and welfare of the Universities of Leiden and Amsterdam.

Quantitative morphological analysis: Golgi-Cox method. Adult rat offspring (low LG, $n=6$; high LG, $n=7$ ) were used for morphological analysis of CA1 pyramidal cells using the Golgi-Cox method as described previously (Boekhoorn et al., 2006). Briefly, animals were decapitated between 8:00 and 9:00 A.M. Trunk blood samples were collected immediately after decapitation and processed for plasma CORT levels using an $\mathrm{I}^{125}$-CORT radioimmunoassay for rats (MP Biomedicals) as described previously (Dalm et al., 2005). The brains were removed and placed in vials containing Golgi-Cox solution and stored in the dark for $30 \mathrm{~d}$. Brains were rinsed in milli-Q water, dehydrated in ethanol, embedded in celloidine, and immersed in chloroform for a maximum of $16 \mathrm{~h}$. Chloroform was discarded, and brains were subsequently immersed in $70 \%$ ethanol. Brains were sliced on a vibratome at a thickness of $100 \mu \mathrm{m}$. Sections were stained using previously described procedures (van Pelt et al., 2005) and mounted on glass slides. Slides were allowed to dry for 2 weeks before initiating the image analysis.

The length and complexity of apical and basal dendrites was determined from five pyramidal cells per rat. The cells were randomly chosen, alternating between right and left hemispheres, in the dorsal hippocampus and had to be clearly filled and free of neighboring precipitate and damage. Spine density was determined in two $20 \mu \mathrm{M}$ segments from the apical and one $20 \mu \mathrm{M}$ segment from the basal dendrite for each cell. Analyses were performed by individuals unaware of the animal's phenotype using a combination of Neurodraw and Image Pro software (van Pelt et al., 2005; Boekhoorn et al., 2006). Values obtained from each group of five cells/individuals were averaged, yielding a single value per animal for the statistical analysis of each measurement category.

Western blot analysis. Hippocampi were dissected from adult animals for Western blotting (low LG, $n=6$; high LG, $n=6$ ). Briefly, hippocampi were homogenized in phosphate buffer $\left(10 \mathrm{mM} \mathrm{KPO}_{4} / 10 \mathrm{~mm}\right.$ $\mathrm{KCl}, \mathrm{pH}$ 7.4), and protein concentration was determined using the Bradford method (BCA protein assay reagent; Pierce Biotechnology). A quantity of $50 \mu \mathrm{g}$ of hippocampal tissue per sample was loaded and electrophoresed on a $4-12 \%$ Bis-Tris gel (Invitrogen) at $200 \mathrm{~V}$ for $60 \mathrm{~min}$ in Tris-glycine running buffer ( $16.5 \mathrm{~mm}$ Tris, $0.135 \mathrm{~m}$ glycine, $0.1 \% \mathrm{SDS}, \mathrm{pH}$ 8.3) and transferred on polyvinylidene difluoride (PVD) membranes (GE Healthcare) at $125 \mathrm{mV}$ overnight at $4^{\circ} \mathrm{C}$. The PVD membranes were incubated in a blocking solution (TBS, pH 7.5, with 5\% elk milk) for 60 $\mathrm{min}$. The blots were then incubated for $60 \mathrm{~min}$ at room temperature with appropriate dilutions of the primary antibody: 1:500 for GR (rabbit, H-300; Santa Cruz Biotechnology), 1:500 for MR (mouse clone 1D5; kindly donated by E. Gomez-Sanchez, Harry S. Truman Memorial VA, Columbia, MO). The blots were washed in TBS buffer and then incubated for $60 \mathrm{~min}$ at room temperature with the appropriate secondary antibodies (goat anti-rabbit HRP, 1:5000, and anti-mouse HRP, 1:5000; Calbiochem). The blots were developed using Western Blot Enhanced Luminol Reagent (ECL) kit (PerkinElmer) and exposed to x-ray film. The films were developed, and intensity of the immunoreactive labeling was analyzed using an image analysis system (NIH ImageJ). Optical density measurements were normalized using $\alpha$-tubulin to control for the amount of protein loaded on the gel. Therefore, each blot was stripped in $0.2 \mathrm{M} \mathrm{NaOH}$ for $20 \mathrm{~min}$ at room temperature and reprobed with an anti- $\alpha$-tubulin antibody (1:1000 mouse; Biodesign International).

Electrophysiology: assessment of long-term potentiation. Adult rat offspring of low $(n=10)$ and high $(n=11)$ mothers were decapitated between 8:00 and 9:00 A.M. Trunk blood samples were collected immediately after decapitation and processed for plasma CORT levels using an $\mathrm{I}^{125}$-corticosterone radioimmunoassay for rats (MP Biomedicals) (Dalm et al., 2005). Briefly, the brain was rapidly removed from the skull and stored in chilled artificial CSF (aCSF) equilibrated with $95 \% \mathrm{O}_{2}$ and $5 \%$ $\mathrm{CO}_{2}$. The aCSF contained the following (in $\mathrm{mM}$ ): $120 \mathrm{NaCl}, 3.5 \mathrm{KCl}, 1.3$ $\mathrm{MgSO}_{4}, 1.25 \mathrm{NaH}_{2} \mathrm{PO}_{4}, 2.5 \mathrm{CaCl}_{2}, 10 \mathrm{D}$-Glucose, and 25.0 $\mathrm{NaHCO}_{3}$. Brain hemispheres were isolated and hippocampi dissected to generate coronal slices ( $\sim 400 \mu \mathrm{m}$ thick) using a manual tissue chopper. The slices were then transferred to a storage bath and allowed to equilibrate for $1 \mathrm{~h}$ at room temperature in oxygenated aCSF (Wiegert et al., 2006).

Slices were transferred into a slice chamber where they were kept submerged in aCSF at a temperature of $31.5^{\circ} \mathrm{C}$. The aCSF bath was refreshed at a rate of $2.5 \mathrm{ml} / \mathrm{min}$ and equilibrated with $95 \% \mathrm{O}_{2}$ and $5 \% \mathrm{CO}_{2}$. Bipolar stimulation electrodes ( $60 \mu \mathrm{m}$ stainless-steel wires insulated except for the tip) were placed on the Schaffer collaterals, and glass recording pipettes (filled with aCSF) were positioned in the CA1 stratum radiatum to record field EPSPs (fEPSPs). At the start of the experiment, an input-output curve was established for the slope of the fEPSP, from which half-maximal stimulation intensity was determined (Wiegert et al., 2006). This intensity was used throughout the remainder of the recording session. After establishing the input-output curve, we moni- 
tored baseline synaptic transmission using half-maximal stimulation intensity with a frequency of $0.017 \mathrm{~Hz}$. In some cases, a population spike superimposed on the fEPSP was seen when stimulating at half-maximal stimulation intensity. In these cases, we reduced the stimulation intensity to spike threshold level for the population spike. When signals were stable during a baseline period of $20 \mathrm{~min}$, repetitive tetanic stimulations ( $10 \mathrm{~Hz} ; 900$ pulses) were applied, after which recording proceeded for another $60 \mathrm{~min}$ at a frequency of $0.017 \mathrm{~Hz}$ (Mayford et al., 1996). CORT (Sigma-Aldrich) treatment was implemented as follows: hippocampal slices were incubated with a high dose of CORT (100 nM dissolved in $0.01 \% \mathrm{ETOH}$ ) for a period of $20 \mathrm{~min}$ and then rinsed with aCSF for a minimum of $60 \mathrm{~min}$ before onset of electrophysiological recordings. Vehicle treatment (VEH; $0.01 \% \mathrm{ETOH}$ in aCSF) was performed in the same manner with the exception that no CORT was added to the medium.

Previous studies in our laboratory demonstrated that effects exerted by CORT are dose dependent (Joels, 2006). Low doses of CORT (1 nM) mimic basal plasma CORT levels and predominantly activate MRs, whereas higher doses (30-100 nM) mimic stress-induced CORT levels and activate GR in addition to MR. High doses (30-100 nM) also allowed the development of effects requiring homodimerization of the GR, which were comparable with those seen after in vivo stress exposure (Karst et al., 2000; Alfarez et al., 2002, 2003). Based on these findings, the high dosage $(100 \mathrm{~nm})$ used in the current study mimicked physiological, stressinduced CORT levels. Perhaps most importantly, the $100 \mathrm{~nm}$ concentration mimics the levels of free CORT during a moderate stressor (e.g., 20 min of restraint) in the rat. Therefore, the concentration is physiologically relevant.

Contextual fear conditioning. Contextual fear conditioning was examined in adult low LG and high LG offspring to assess the effect of maternal care on hippocampal-dependent learning during conditions of high stress. Contextual fear conditioning is a learning task that involves hippocampal as well as amygdala processing, and the aversive nature of the shock stimulus makes the task inherently highly stressful (Phillips and LeDoux, 1992; Maren, 2001).

Animals were habituated to manipulations associated with testing for $3 \mathrm{~d}$ before the conditioning session. Each day, animals were transported to the testing room and briefly handled by the experimenter. On the conditioning day, animals were transported to the testing room and placed in the conditioning chamber. The conditioning chamber consisted of a plastic box with a clear plastic top that was placed inside a wooden sound-attenuating box containing a $40 \mathrm{~W}$ light bulb and a ventilation fan mounted inside. The front of each wooden box was left open to allow visualization by a video camera. The rod floors were wired to a shock generator and scrambler to deliver a $1 \mathrm{~s}, 1 \mathrm{~mA}$ shock, which has been demonstrated to induce a significant rise in circulating CORT levels (Cordero et al., 2003). Disposable cardboard was placed beneath the conditioning chamber and, after each animal, the cardboard was replaced and the chamber cleaned with 5\% ethanol. On the day of conditioning, animals were tested in two different versions of the task: hippocampal dependent and hippocampal independent. In the hippocampal-dependent version, animals (low LG, $n=6$; high LG, $n=$ 7) were allowed to explore the context for $120 \mathrm{~s}$ before receiving a single foot shock, after which animals remained in the context an additional $30 \mathrm{~s}$ before removal to the home cage. This procedure allowed sufficient time for rats to explore and form a hippocampal representation of context. In the hippocampal-independent version, animals (low LG, $n=6$; high LG, $n=8$ ) were not allowed to explore the context but rather received a shock immediately after placement in the context allowing insufficient time for rats to form a hippocampal representation of context. Under these circumstances, the hippocampal contribution to conditioning has been shown to be minimized (Kiernan and Westbrook, 1993; Westbrook et al., 1994) .

The animals were returned to the conditioning context the next day ( $24 \mathrm{~h}$ later) to assess contextual fear memory in a 4 min test, during which no shocks were administered. Freezing, characterized as the absence of all body movements except those required for respiration (Blanchard and Blanchard, 1969), was scored as an index of contextual fear learning. Following a time-sampling method, every $3 \mathrm{~s}$ an observation was made, and the presence or absence of freezing was noted. A percentage score was calculated for the proportion of observation periods in which freezing was observed.

Statistical analysis. Statistical analyses were performed using SPSS for Windows (version 12.0.1). Graphs were plotted using Prism Graph Pad software. Quantitative morphological analysis for dendritic length and spine densities, pre-LTP recording electrophysiological properties, Western blot, maternal behaviors cumulated over 1 week, and endocrine measurements were performed using unpaired (two-tailed) Student's $t$ test. Two-way ANOVA with maternal care (low LG and high LG) as a between subjects factor and observation day (days 1-6) as a within subjects factor was used to analyze percentage of maternal LG across day. Mauchly's test of sphericity was applied and the degrees of freedom (df) corrected to more conservative values using the Huynh-Feldt (H-F) $\varepsilon(\varepsilon)$ if the assumption of sphericity was violated. Significant main effects of observation day were further decomposed using pairwise comparisons with a Sidak correction.

Two-way-ANOVA with maternal care (low and high LG) as a between subjects factor and preshock interval $(0,120)$ or treatment (VEH, CORT) as a within subjects factor was used for analysis of fEPSP (averaged over the last $20 \mathrm{~min}$ of recording) and contextual freezing data. Significant main effects of maternal care were further decomposed using pairwise comparisons with a Sidak post hoc and Bonferroni's correction, respectively, for multiple comparisons. Data are presented as mean \pm SEM, and probability level of $5 \%$ was used as the minimal criterion of significance.

\section{Results}

\section{Maternal care characterization}

The frequency of the maternal behaviors is listed in Table 1. In addition to differing in the percentage of LG averaged over the first week of life, dams used in the current study also differed in the percentage of flat-back nursing (a form of passive maternal care) with low LG dams displaying significantly more flat-back nursing than high LG dams $\left(t=2.78 ; \mathrm{df}_{(30)}, p<0.01\right.$, unpaired $t$ test). High LG dams displayed significantly more LG than low LG dams across all days of the observation period $\left(F_{(1,21)}=145.11\right.$; $p<0.0001$, two-way ANOVA, repeated measures) (Fig. $1 B$ ). Furthermore, the frequency of maternal LG decreased over days across both groups (uncorrected, $F_{(5,105)}=3.10, p<0.05 ; \mathrm{H}-\mathrm{F}$ corrected, $\left.F_{(3.8,79.7)}=3.10 ; \varepsilon=0.759 ; p<0.05\right)$. These findings are in agreement with previous reports and support the view that, in general, low LG dams display a more passive maternal care style than high LG dams (Champagne et al., 2003; Menard and Hakvoort, 2007).

\section{Maternal care induces morphological changes in CA1 neurons: evidence for experience-dependent plasticity} Enduring effects of variations in maternal care on hippocampal development have been suggested to occur via mechanisms similar to those associated with experience-dependent neural development (for review, see Meaney, 2001). This raises the question of whether early sensory experiences such as maternal LG (a form of tactile stimulation) could stably alter dendritic morphology and/or spine density during a critical period of development. To address this question, we used Golgi staining, a sensitive method allowing accurate assessment of morphological aspects of hippocampal neurons. We report morphological alterations in the length of both basal ( $t=3.056 ; \mathrm{df}=11 ; p<0.01$, unpaired $t$ test $)$ and apical $(t=2.38$; df $=11 ; p<0.05$, unpaired $t$ test $)$ CA1 pyramidal cell dendrites that were significantly shorter in adult offspring of low versus high LG mothers (Fig. 2 A, Table 2). Spine density (as determined in $20 \mu \mathrm{m}$ segments) of apical $(t=2.88$; $\mathrm{df}=11 ; p<0.05$, unpaired $t$ test $)$ and basal $(t=2.57$; $\mathrm{df}=11$; $p<0.05$, unpaired $t$ test) dendrites was also significantly lower in low versus high LG offspring (Fig. $2 B$, Table 2). Although no 
Scoring every 3 minutes

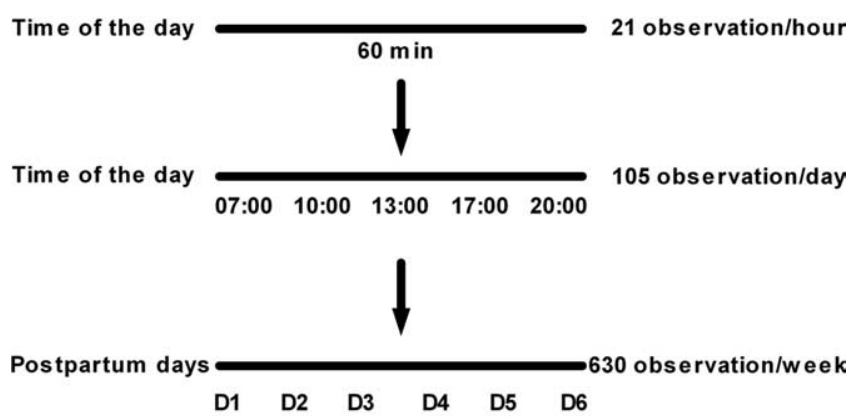

B

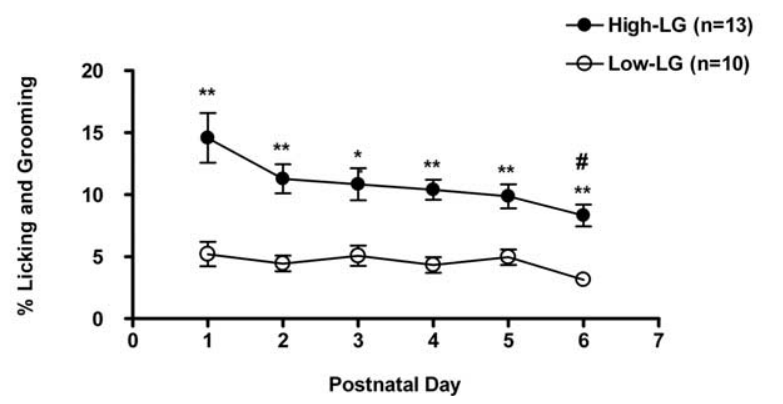

Figure 1. Maternal behavior characterization. $A, A$ total of five maternal observation periods per day [7:00 A.M. (07:00), 10:00 A.M. (10:00), 1:00 P.M. (13:00), 5:00 P.M. (17:00), and 8:00 P.M. (20:00)] were conducted from P1 (day of birth, P0) until P6 (D1-D6). Each observation period lasted $60 \mathrm{~min}$ and contained 21 observations, which were spaced by a 3 min interval. At the end of the $6 \mathrm{~d}$ observation period, a total of 630 observations were cumulated for each dam. $B$, Percentage of maternal $L G$ across days. Low $L G$ and high $L G$ dams differed based on the amount of $L G$ provided to their pups during the first week postpartum. Compared with low $L G$ dams, the percentage of $L G$ was consistently greater in high $L G$ dams on each day. Note that $L G$ scores significantly decreased over days for both high $\mathrm{LG}$ and low $\mathrm{LG}$ dams. ${ }^{*} p<0.05$, ${ }^{* *} p<$ 0.01 , compared with low $L G$ dams; $\# p<0.05$, compared with values on day 1 within subject.

significant differences were observed in the number of dendritic branches (data not shown) as a function of maternal care, a trend toward statistical significance for the number of branching points was observed between offspring of low and high LG mothers, with low LG offspring displaying a trend toward fewer branching points on apical $(t=1.97 ; \mathrm{df}=11, p>0.05$, unpaired $t$ test $)$ but not basal $(t=0.91 ; \mathrm{df}=11 ; p>0.05$, n.s. unpaired $t$ test $)$ dendrites than high LG offspring (Table 2).

\section{Synaptic functioning under resting conditions is greatly altered by history of maternal care}

Previous reports showed that as early as P8, there is decreased expression of genes encoding NMDA receptor (NR) subunits (i.e., NR2A and NR2B, particularly in the CA1 subfield) in the offspring of low compared with high LG mothers (Liu et al., 2000; Bredy et al., 2004). These characteristics endure into adulthood and correlate with impaired cognitive performance in hippocampal-dependent tasks (e.g., Morris water maze, object recognition) (Liu et al., 2000; Bredy et al., 2003). Therefore, we induced tetanic stimulation of Schaffer collaterals in hippocampal slices taken from low and high LG rats, which were bathed with either VEH or CORT and recorded synaptic activity for the next 60 min afterward. We report a significant interaction between maternal care (low versus high LG) and treatment (VEH versus CORT; $F_{(1,8)}=60.31 ; p<0.001$, two-way ANOVA, repeated measure). Sidak post hoc comparisons revealed dramatic differences after tetanic stimulation as a function of maternal care $(p<0.001)$. Although synaptic potentiation was very prominent in rats from high LG mothers, no significant synaptic potentiation was seen 60 min after tetanic stimulation ( $10 \mathrm{~Hz}$; 900 pulses) in offspring from low LG mothers (Fig. 3). It is noteworthy that the maximal responses evoked by low-frequency stimulation were significantly lower in offspring of low LG versus high LG mothers $(t=3.59$; $\mathrm{df}=17 ; p<0.01$, unpaired $t$ test $)$, whereas half-maximum stimulus intensity values were comparable between both groups (Table 3 ). These findings suggest alterations in pre- and post-LTP synaptic transmission properties between the adult offspring of high and low LG mothers and reinforce the suggestion that differential synaptic transmission might be related to differences in morphological aspects of CA1 neurons reported above.

\section{Corticosteroid receptor protein levels are altered by maternal} care without affecting MR/GR ratio

To ascertain that the effects reported above were not attributable to different levels of circulating basal CORT and ACTH, trunk blood samples were taken at rest when animals were killed by decapitation for either Golgi analysis (cohort 1: low LG, $n=6$; high LG, $n=7$ ), glucocorticoid receptor system analysis (cohort 2: low LG, $n=5$; high LG, $n=5$ ), or electrophysiology (cohort 3: low LG, $n=10$; high LG, $n=11$ ). For all cohorts, we report that CORT and ACTH levels did not differ under resting condition between low and high LG offspring. All values for CORT and ACTH levels are reported in Table 4 for each individual cohort.

Stress and high CORT are known modulators of hippocampal functioning during learning and memory. CORT exerts its action via binding to MR and/or GR, which are both abundantly expressed in CA1 pyramidal neurons (de Kloet et al., 1998). Although GR activation mediates the inhibitory effects of high CORT on LTP, conditions of predominant MR activation (resting state) are associated with efficient induction of LTP (Diamond et al., 1992; Pavlides et al., 1996; Joels and Krugers, 2007). Differences in MR and GR protein levels between low and high LG offspring may thus affect LTP, both under resting and stresslike conditions.

We measured protein levels for both MR and GR in the hippocampus of offspring of low and high LG mothers. In agreement with previous reports (Liu et al., 1997; Weaver et al., 2004, 2005), we observed lower GR protein levels in low LG [relative optical density (ROD), $32.85 \pm 1.64$ ] compared with high LG adult offspring $(\mathrm{ROD}, 58.77 \pm 1.75 ; t=10.34$; $\mathrm{df}=10 ; p<0.001$, unpaired $t$ test) (Fig. $4 A$ ). We extended this finding by showing that MR levels are also lower in low LG (ROD, $17.8 \pm 1.48)$ compared with high LG adult offspring (ROD, $33.37 \pm 3.31 ; t=3.68$; $\mathrm{df}=$ $10 ; p<0.01$, unpaired $t$ test) (Fig. $4 B$ ). However, calculation of the MR/GR ratio revealed no differences between low LG and high LG offspring (low LG, $0.54 \pm 0.05$, vs high LG, $0.53 \pm 0.05$ ). The MR/GR ratio was obtained by dividing the ROD value of MR by that of GR for each phenotype.

\section{Corticosterone modulation of LTP is altered by history of maternal care}

Next, we tested whether the differential levels of GR and MR expression reported above are associated with functional effects of high CORT on CA1 neuronal excitability. We pretreated hippocampal slices from adult offspring of low and high LG mothers with stress-like levels of CORT (100 nM) and induced LTP 1-4 h 
A

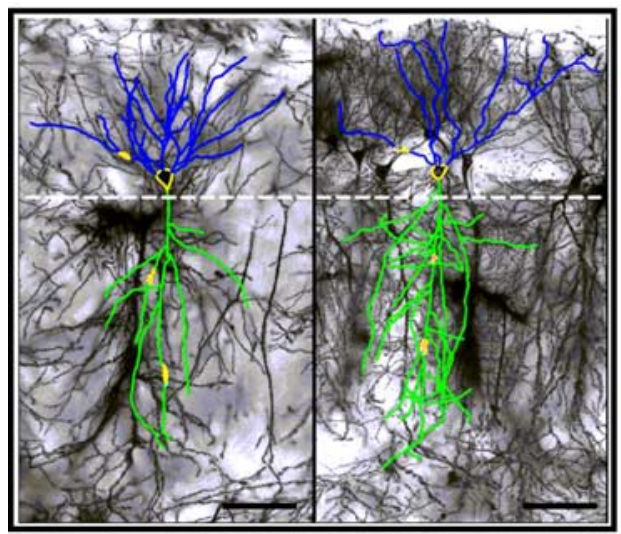

Low LG
B

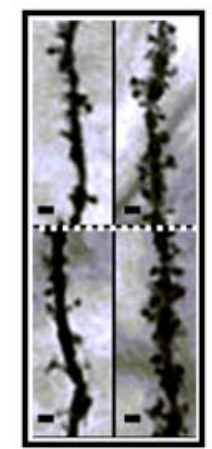

Low LG High LG

Figure 2. Quantitative morphological analysis of dendritic length. $\boldsymbol{A}$, Representative photographs of Golgi-stained CA1 pyramidal neurons accompanied by typical drawing taken from adult rats that received low (left) versus high (right) amounts of $L G$ in infancy. Apical dendrites on top (blue) and basal dendrites at the bottom (green) are delineated by a dotted line. Yellow marks represent typical locations where spine density was assessed. Drawings are based on three-dimensional stacks of Golgi-stained cells allowing accurate distinction between the cell of interest and neighboring cells. Note that the length of dendritic trees (both basal and apical dendrites) is significantly shorter in low $\mathrm{LG}(n=6)$ compared with high $\mathrm{LG}(n=7)$ offspring. Magnification, $40 \times$. Scale bars, $200 \mu \mathrm{m}$. B, Representative photographs depicting spine density for low (left) and high (right) $L G$ offspring. Basal dendrites at the top and apical dendrites at the bottom are delineated by a dotted line. Note that spine density is lower in offspring of low $L G(n=6)$ compared with high LG $(n=7)$ mothers. Magnification, $630 \times$. Scale bars, $2.5 \mu \mathrm{m}$. From each cell, two $20 \mu \mathrm{m}$ segments from the apical dendrite and one $20 \mu \mathrm{m}$ segment from basal dendrite were analyzed (see yellow marks for typical locations in $\boldsymbol{A})$.

later to allow gene-mediated GR effects to develop (Karst et al., 2000). We report a significant interaction between maternal care (low vs high LG) and treatment (VEH vs CORT; $F_{(1,8)}=60.31$; $p<0.001$, two-way ANOVA, repeated measure). Sidak post hoc comparison revealed that compared with VEH conditions, the percentage increase in fEPSP after tetanic stimulation was reduced in slices from high LG offspring pretreated with high CORT $(p<0.01)$. This is in agreement with previous studies using a similar dose of CORT using rats with unknown history of maternal care (Diamond et al., 1992; Pavlides et al., 1996; Wiegert et al., 2005; Joels and Krugers, 2007) (Fig. 5A).

Surprisingly, slices from low LG offspring displayed an enhanced, rather than reduced, LTP in response to a high dose of CORT (Fig. $5 B)\left(F_{(1,8)}=60.38 ; p<0.001\right.$, two-way ANOVA, repeated measures, and $p<0.01$, Sidak post hoc comparison). Moreover, we found that, contrary to what was observed under resting conditions, maximal responses evoked by low-frequency stimulation and half-maximum stimulus intensity values did not differ between low and high LG offspring (Table 3), suggesting that high CORT pretreatment abolished differences in synaptic transmission observed at rest between phenotypes.

Contextual fear conditioning is enhanced in low LG offspring The offspring of high compared with low LG mothers show improved learning/memory in a low to moderately stressful learning context (Bredy et al., 2003). Based on the in vitro electrophysiology findings reported above, we predicted that the low LG offspring would display better memory formation in vivo in a highly stressful learning context. To address this question, we used a contextual fear conditioning task. To assess hippocampal

contribution, we used two versions of the task [i.e., hippocampal independent (short interval between placement in the test box and shock delivery) and hippocampal dependent ( $120 \mathrm{~s}$ interval between placement in test box and administration of shock, allowing the development of a spatial representation)]. Consistent with our hypothesis, we report a significant interaction between maternal care (low LG and high LG) and intervals ( 0 and $120 \mathrm{~s})\left(F_{(4,57)}=4.576 ; p<0.05\right.$, two-way ANOVA). Pairwise comparisons using a Bonferroni's adjustment for multiple comparisons revealed that low LG rats showed enhanced freezing (i.e., greater contextual fear learning) in the hippocampal-dependent contextual fear memory test relative to high $\mathrm{LG}$ rats (120 s preshock interval). No effect of maternal care was seen when hippocampal processing was prevented by an immediate shock ( $0 \mathrm{~s}$ preshock interval) (Fig. 6).

\section{Discussion}

Variations in postpartum maternal care were associated with effects on morphology, synaptic functioning, CORT responsiveness of CA1 neurons, and hippocampaldependent learning in a stressful context. The adult offspring of high LG mothers exhibited longer dendritic branch length and increased spine density in CA1 neurons, alterations in electrophysiological properties at rest, enhanced LTP, and increased hippocampal expression of both MR and GR, with no change in MR/GR ratio. Exposure to stress-like levels of CORT impaired LTP in the adult offspring of high LG mothers. In contrast, the same dose of CORT greatly enhanced LTP in low LG offspring. A comparable effect was observed in vivo. The offspring of low LG mothers displayed enhanced hippocampal-dependent learning under stressful conditions.

\section{Maternal care, CA1 neuronal morphology, and synaptic function}

Previous studies suggest effects of early experience on neuronal morphology and synaptic function in the hippocampus (Poeggel et al., 2003; Ovtscharoff et al., 2006), including effects of postnatal "handling" on hippocampal LTP (Wilson et al., 1986; Tang and Zou, 2002). Such effects occur during hippocampal maturation (Pokorny and Yamamoto, 1981; Turner et al., 1998). Although migration of pyramidal neurons is complete at birth, there is a burst of postnatal growth that includes increases in synapse and spine number (Turner et al., 1998). These events occur during intense periods of mother-infant interactions and may thus be regulated by variations in maternal care. Increased pup LG in the rat is associated with enhanced expression of NR2A and NR2B subunits of the NMDA receptor as well as neurotrophic factors and may have therefore created an environment favorable for synaptic growth (Liu et al., 2000; Bredy et al., 2003, 2004). We speculate that such maternal effects could include differential innervation along the dendritic tree, thus promoting altered processing of information at the synapse. Morphological changes could account for the observed alteration in synaptic functioning and ultimately contribute to the previously reported phenotypic differences in hippocampal-dependent learning under low-stress conditions, such as object recognition or spatial learning (Liu et al., 2000; Bredy et al., 2003). Whether such maternally induced changes in CA1 neuronal morphology are stable over time remains to be demonstrated. However, enduring changes (up to $45 \mathrm{~d}$ ) in the cingulate cortex after cessation of repeated maternal 
separation supports the possibility of stability into adulthood (Helmeke et al., 2001).

\section{Corticosterone modulation of LTP and maternal care}

We observed prominent LTP in offspring of high LG mothers under basal conditions. Pretreatment with a high dose of CORT strongly impaired subsequent induction of LTP in high LG offspring, consistent with previous reports (Wiegert et al., 2005; Joels and Krugers, 2007). Unexpectedly, whereas the low LG offspring displayed poor LTP under basal conditions, we observed that pre-exposure to high CORT resulted in a large enhancement of LTP compared with no-CORT conditions.

Interestingly hippocampal GR expression differs markedly between the two phenotypes. Compared with low LG, high LG offspring display increased hippocampal GR expression, a phenomenon accounted for by epigenetic modifications (Weaver et al., 2004). We also found increased hippocampal MR expression in high compared with low LG offspring, suggesting that although the ratio between MR and GR is unchanged, the relative amount of both receptor types differs significantly. Reduced MR and GR levels could lead to a distinct functional outcome with regard to hippocampal cell excitability (de Kloet et al., 1998).

Although predominant MR activation (under resting conditions) is associated with efficient induction of LTP, additional GR activation (under stress conditions) leads to impaired LTP induction (Diamond et al., 1992; Pavlides et al., 1996; Joels and Krugers, 2007). Consequently, under resting conditions (low CORT, predominant MR activation), lower levels of MR in combination with poorer dendritic morphology may contribute to impaired LTP in low relative to high LG offspring. Conversely, under highly stressful conditions (high CORT, additional GR activation), lower levels of GR might drastically reduce or delay the suppressive effects of GR-mediated genomic events in low relative to high LG offspring. Clearly, we cannot exclude that other changes in the GR (or MR) signaling pathways may underlie the observed dichotomy in cellular responses to CORT. Interestingly, such a dichotomy in responsivity to CORT has been described previously, where exposure to stress or CORT before training in the Morris water maze impaired learning in wild-type mice but facilitated learning in the apolipoproteinE knock-out mice (Grootendorst et al., 2001).

We acknowledge the possibility of a relatively rapid neuronal remodeling in response to high CORT in our slice preparation, which in turn could contribute to the effects on LTP. Such a possibility has been observed before by our group where exposure to high CORT was able to induce rapid morphological changes in slices from handled rats (reversed by GR antagonism in vivo) (Alfarez et al., 2008). Interestingly, this phenomenon seems to be dependent on previous life history, because exposure to high CORT in vitro does not result in changes in morphology in naive rats (Alfarez et al., 2008). Together, these findings suggest that life history (e.g., handling or maternal care), which results in changes in MR and/or GR levels, may act as a determining factor of the effects of high CORT on cell function and morphology. The results of the present study represent a clear example of this phenomenon. However, whether changes in morphology occur and underlie differential LTP and behavior in response to high CORT remains to be addressed by future studies.

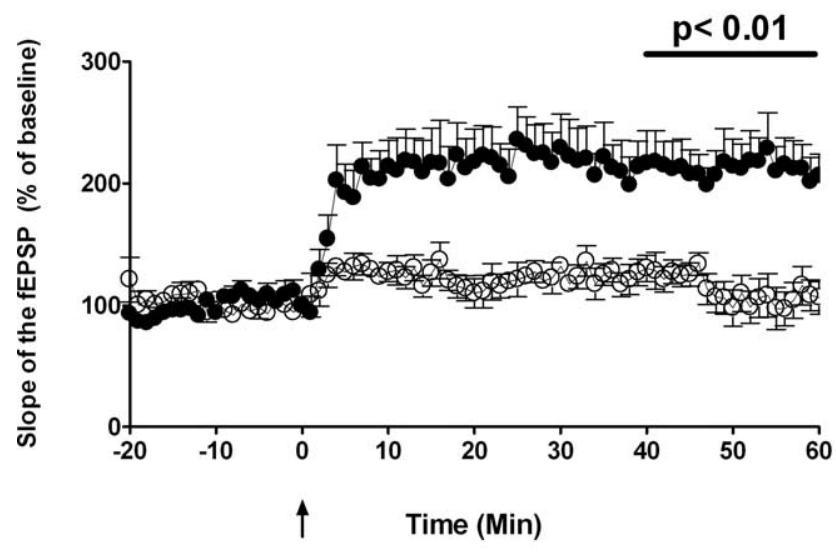

Tetanic Stimulation

Figure 3. Synaptic potentiation is impaired in low versus high licking and grooming offspring under basal conditions. Tetanic stimulation ( $10 \mathrm{~Hz}, 900$ pulses) of Schaffer collateral afferents significantly potentiated the slope of the fEPSP in high $(n=11)$ but not low $(n=10)$ $\mathrm{LG}$ offspring. The bar represents the last $20 \mathrm{~min}$ of recording.

\section{Maternal care and learning in a highly stressful context}

The adult offspring of high LG mothers show enhanced hippocampal-dependent learning (object recognition) under low-stress conditions (Bredy et al., 2003, 2004). In contrast, under stressful conditions (foot shock), we found enhanced hippocampal-dependent learning in the offspring of low compared with high LG offspring. Therefore, the low relative to high LG offspring showed increased conditioned freezing in a hippocampal-dependent version of a fear-conditioning task. These findings support our in vitro prediction that low, but not high, LG offspring would display enhanced memory formation under stressful conditions.

The mechanism for this difference in contextual fear conditioning remains to be clearly defined. Previous studies suggest that CORT might facilitate contextual fear conditioning (Cordero et al., 1998). Moreover, the offspring of low LG mothers show increased CORT responses to acute stress (Liu et al., 1997). Therefore, the differences in performance in the contextual fear conditioning test might occur as a result of higher levels of CORT in the low LG offspring, processes underlying improved hippocampal LTP in response to high levels of CORT as demonstrated in the current studies, or a combination of the two. Cordero et al. (1998) show evidence for a relationship between plasma CORT and freezing in a contextual fear-conditioning paradigm, but whereas freezing levels in this study increased significantly with shock intensity (i.e., $0.4-1.0 \mathrm{~mA}$ ), there was no correspond- 
Table 3. Electrophysiological properties of CA1 hippocampal neurons (pre-LTP recordings)

\begin{tabular}{lcc}
\hline & Low LG $(n=10)$ & High LG $(n=11)$ \\
\hline Vehicle treatment & & $-2.98 \pm 0.17^{* *}$ \\
Max fEPSP amplitude $(\mathrm{mV})$ & $-2.16 \pm 0.19$ & $216 \pm 4.82$ \\
Max stimulus intensity & $211 \pm 7.36$ & \\
100 nм Corticosterone treatment & & $-2.64 \pm 0.09$ \\
Max fEPSP amplitude $(\mathrm{mV})$ & $-2.56 \pm 0.33$ & $216 \pm 4.64$ \\
Max stimulus intensity & $203 \pm 8.47$ & \\
\hline
\end{tabular}

Pre-LTP recording measurements. Maximal fEPSP amplitude evoked by low-frequency stimulation was significantly lower in offspring of low $L G$ versus high $\mathrm{LG}$ mothers, whereas half-maximum stimulus intensity values were comparable between both groups in response to vehicle treatment. Conversely, maximal fEPSP amplitude evoked by low-frequency stimulation and half-maximum stimulus intensity values did not differ between low and high LG phenotypes after high corticosterone $(100 \mathrm{~nm})$ treatment. ${ }^{* *} p<0.01$.

Table 4. Endocrine measurements

\begin{tabular}{ccc}
\hline & Low LG & High LG \\
\hline Cohort 1 & $n=6$ & $n=7$ \\
ACTH $(\mathrm{pg} / \mathrm{ml})$ & $210.7 \pm 49.9$ & $189.5 \pm 55.5$ \\
CORT $(\mathrm{ng} / \mathrm{ml})$ & $15.2 \pm 7.4$ & $12.3 \pm 8.9$ \\
Cohort 2 & $n=5$ & $n=5$ \\
ACTH $(\mathrm{pg} / \mathrm{ml})$ & $204.6 \pm 53.4$ & $194.4 \pm 61.1$ \\
CORT $(\mathrm{ng} / \mathrm{ml})$ & $18.4 \pm 11.1$ & $14.5 \pm 10.9$ \\
Cohort 3 & $n=10$ & $n=11$ \\
ACTH $(\mathrm{pg} / \mathrm{ml})$ & $209.6 \pm 54.7$ & $193.5 \pm 66.6$ \\
CORT $(\mathrm{ng} / \mathrm{ml})$ & $18.2 \pm 9.3$ & $13.4 \pm 9.8$ \\
\hline
\end{tabular}

Mean ( \pm SEM) endocrine measurements. CORT and ACTH levels did not differ under resting condition between low and high LG phenotypes for the cohorts used in the current study to determine hippocampal properties. Cohort 1 (used for Golgi analysis; low $L G, n=6$; high $L G, n=7$ ), cohort 2 (used for corticosteroid receptor analysis; low $\mathrm{LG}, n=5$; high $\mathrm{LG}, n=5$ ), or cohort 3 (used for electrophysiology; low $\mathrm{LG}, n=10$; high $\mathrm{LG}, n=11$ ).

A

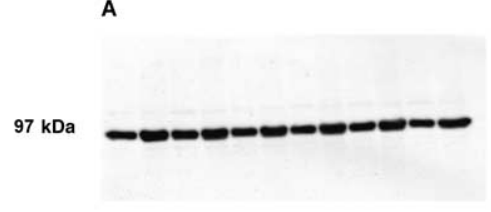

$55 \mathrm{kDa}$
B

$107 \mathrm{kDa}$

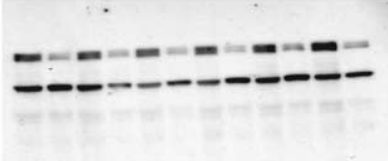

$55 \mathrm{kDa}$

H L H L H L H L H L H L

Figure 4. The corticosteroid receptor protein levels. $A$, Representative photograph of a GR Western blot normalized against $\alpha$-tubulin. GR protein levels were found to be significantly reduced in low $(n=6) \mathrm{LG}$ compared with high $(n=6) \mathrm{LG}$ offspring. $\boldsymbol{B}$, Representative photograph of a MR Western blot normalized against $\alpha$-tubulin. MR protein levels were also found to be significantly decreased in low $L G$ compared with high $L G$ offspring. Note that the secondary band underneath represents nonspecific signal. L, Low LG offspring; H, high LG offspring. GR, 97 kDa; MR, 107 kDa; $\alpha$-tubulin, 55 kDa.

ing increase in plasma CORT. Pugh et al. (1997) showed an increase in freezing in a contextual fear-conditioning test associated with CORT administration that mimics postshock CORT levels (i.e., $0.25-1.00 \mathrm{mg} / \mathrm{kg}$ ). The maximal CORT effect was apparent at the low $(0.25 \mathrm{~m} / \mathrm{kg})$ dose, suggesting that CORT levels are permissive for contextual fear conditioning, but that variations within the range of very high levels of CORT may not determine differences in the acquisition of the contextually conditioned freezing (Pugh et al., 1997). Nevertheless, the possible influence of differences in CORT levels between the offspring of high and low LG mothers should be considered in future studies.

What we suggest from the current data is that the differences in behavior are consistent with the differential effects of CORT on hippocampal synaptic plasticity. Furthermore, high levels of CORT and subsequent MR- and GR-mediated genomic events that follow shock exposure could modulate the difference in strength/formation of memory for contextual fear conditioning between phenotypes. This could be accomplished in the current study by the apparent differential sensitivity of hippocampal neu- rons to CORT (presumably conferred through differential hippocampal MR and GR driven responses) between phenotypes. Although in vivo stressful conditions involve the activation of multiple hormone systems in addition to CORT, it is nevertheless striking to observe that maternal care induces such similarity between the in vitro effects of CORT on LTP and the in vivo effects of stress on memory formation.

There is a sparse literature on how electrophysiology (e.g., LTP) can accurately predict behavior in living animals. Examples of successful prediction can be appreciated from recent studies showing that hippocampal LTP occurs during hippocampal learning (Whitlock et al., 2006) in behaving animals. In the current study, our findings that LTP can predict behavior in living animals performing a hippocampal-dependent task under stress provide an additional example of successful prediction from in vitro to in vivo.

In conclusion, the results demonstrate the impact of maternal care on structure and function of the CA1 neural network in offspring and support the view that the early rearing environment may modulate hippocampal development and function. Under certain conditions (decreased pup LG), such effects may actually enhance learning and memory processes under stressful conditions, suggesting that individual differences in outcome of early experience depend on environmental context in later life.

\section{References}

Alfarez DN, Wiegert O, Joels M, Krugers HJ (2002) Corticosterone and stress reduce synaptic potentiation in mouse hippocampal slices with mild stimulation. Neuroscience 115:1119-1126.

Alfarez DN, Joels M, Krugers HJ (2003) Chronic unpredictable stress impairs long-term potentiation in rat hippocampal CA1 area and dentate gyrus in vitro. Eur J Neurosci 17:1928-1934.

Alfarez DN, Karst H, Velzing EH, Joels M, Krugers HJ (2008) Opposite effects of glucocorticoid receptor activation on hippocampal CA1 dendritic complexity in chronically stressed and handled animals. Hippocampus 18:20-28.

Blanchard RJ, Blanchard DC (1969) Crouching as an index of fear. J Comp Physiol Psychol 67:370-375.

Boekhoorn K, Terwel D, Biemans B, Borghgraef P, Wiegert O, Ramakers GJ, de Vos K, Krugers H, Tomiyama T, Mori H, Joels M, Van LF, Lucassen PJ (2006) Improved long-term potentiation and memory in young $\tau$-P301L transgenic mice before onset of hyperphosphorylation and tauopathy. J Neurosci 26:3514-3523.

Bredy TW, Humpartzoomian RA, Cain DP, Meaney MJ (2003) Partial reversal of the effect of maternal care on cognitive function through environmental enrichment. Neuroscience 118:571-576.

Bredy TW, Zhang TY, Grant RJ, Diorio J, Meaney MJ (2004) Peripubertal environmental enrichment reverses the effects of maternal care on hippocampal development and glutamate receptor subunit expression. Eur J Neurosci 20:1355-1362.

Bremner JD, Narayan M, Anderson ER, Staib LH, Miller HL, Charney DS (2000) Hippocampal volume reduction in major depression. Am J Psychiatry 157:115-118.

Buss C, Lord C, Wadiwalla M, Hellhammer DH, Lupien SJ, Meaney MJ, Pruessner JC (2007) Maternal care modulates the relationship between 
A

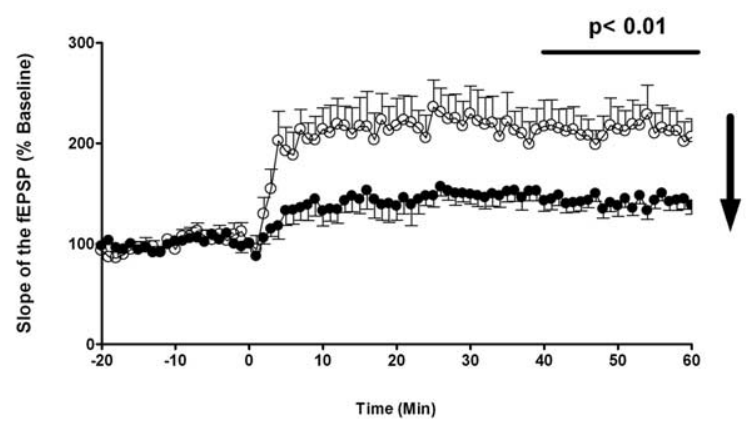

B

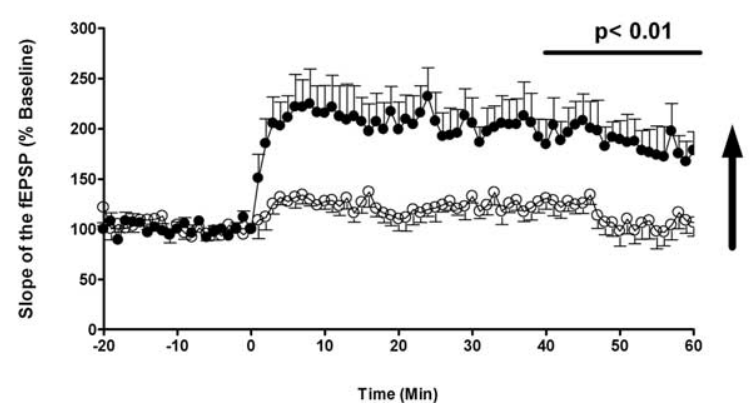

Figure 5. Corticosteroid modulation of LTP. A, Compared with VEH conditions, a significant reduction in the percentage of fEPSPs was prominent in slices from high $L G$ offspring pretreated with $100 \mathrm{~nm}$ CORT. $\boldsymbol{B}$, In contrast, slices from low $\mathrm{LG}$ offspring did not display reduced but rather enhanced LTP in response to a high dose of CORT. The bar represents the last 20 min of recording.

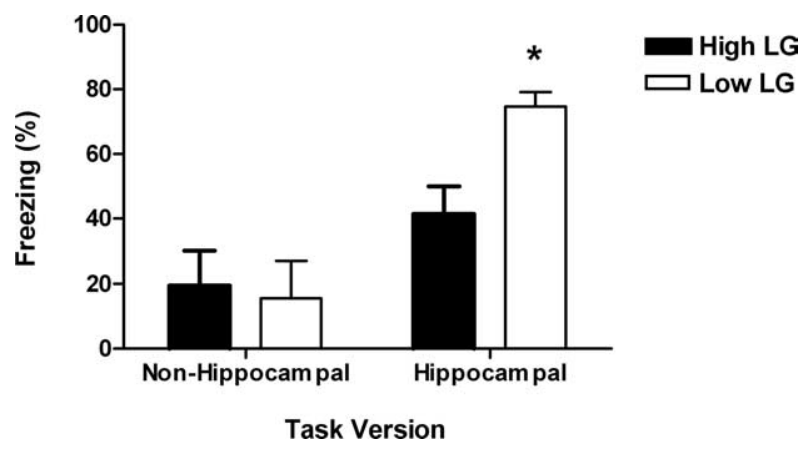

Figure 6. Contextual fear conditioning. When contextual fear learning was assessed $24 \mathrm{~h}$ after conditioning, low $L G$ animals showed significantly greater freezing in the conditioned context than high $L G$ animals in the hippocampal-dependent version of the task (i.e., the situation where animals were allowed to form a spatial representation of the shock environment). However, maternal care did not influence the levels of freezing observed among animals conditioned in the hippocampal-independent/brief preshock interval version of the task $\left({ }^{*} p<\right.$ 0.05).

prenatal risk and hippocampal volume in women but not in men. J Neurosci 27:2592-2595.

Caldji C, Tannenbaum B, Sharma S, Francis D, Plotsky PM, Meaney MJ (1998) Maternal care during infancy regulates the development of neural systems mediating the expression of fearfulness in the rat. Proc Natl Acad Sci USA 95:5335-5340.

Caldji C, Diorio J, Meaney MJ (2003) Variations in maternal care alter GABA(A) receptor subunit expression in brain regions associated with fear. Neuropsychopharmacology 28:1950-1959.
Champagne FA, Francis DD, Mar A, Meaney MJ (2003) Variations in maternal care in the rat as a mediating influence for the effects of environment on development. Physiol Behav 79:359-371.

Coldwell J, Pike A, Dunn J (2006) Household chaos-links with parenting and child behaviour. J Child Psychol Psychiatry 47:1116-1122.

Cordero MI, Merino JJ, Sandi C (1998) Correlational relationship between shock intensity and corticosterone secretion on the establishment and subsequent expression of contextual fear conditioning. Behav Neurosci 112:885-891.

Cordero MI, Venero C, Kruyt ND, Sandi C (2003) Prior exposure to a single stress session facilitates subsequent contextual fear conditioning in rats. Evidence for a role of corticosterone. Horm Behav 44:338-345.

Dalm S, Enthoven L, Meijer OC, van der Mark MH, Karssen AM, de Kloet ER, Oitzl MS (2005) Age-related changes in hypothalamic-pituitaryadrenal axis activity of male C57BL/6J mice. Neuroendocrinology 81:372-380.

de Kloet ER, Vreugdenhil E, Oitzl MS, Joels M (1998) Brain corticosteroid receptor balance in health and disease. Endocr Rev 19:269-301.

Diamond DM, Bennett MC, Fleshner M, Rose GM (1992) Inverted-U relationship between the level of peripheral corticosterone and the magnitude of hippocampal primed burst potentiation. Hippocampus 2:421-430.

Diorio J, Meaney MJ (2007) Maternal programming of defensive responses through sustained effects on gene expression. J Psychiatry Neurosci 32:275-284.

Francis D, Diorio J, Liu D, Meaney MJ (1999) Nongenomic transmission across generations of maternal behavior and stress responses in the rat. Science 286:1155-1158.

Grootendorst J, de Kloet ER, Dalm S, Oitzl MS (2001) Reversal of cognitive deficit of apolipoprotein $\mathrm{E}$ knockout mice after repeated exposure to a common environmental experience. Neuroscience 108:237-247.

Helmeke C, Ovtscharoff Jr W, Poeggel G, Braun K (2001) Juvenile emotional experience alters synaptic inputs on pyramidal neurons in the anterior cingulate cortex. Cereb Cortex 11:717-727.

Joels M (2006) Corticosteroid effects in the brain: U-shape it. Trends Pharmacol Sci 27:244-250.

Joels M, Krugers HJ (2007) LTP after stress: up or down? Neural Plast 93202.

Karst H, Karten YJ, Reichardt HM, de Kloet ER, Schutz G, Joels M (2000) Corticosteroid actions in hippocampus require DNA binding of glucocorticoid receptor homodimers. Nat Neurosci 3:977-978.

Kendler KS, Gardner CO, Prescott CA (2002) Toward a comprehensive developmental model for major depression in women. Am J Psychiatry 159:1133-1145.

Kiernan MJ, Westbrook RF (1993) Effects of exposure to a to-be-shocked environment upon the rat's freezing response: evidence for facilitation, latent inhibition, and perceptual learning. Q J Exp Psychol B 46:271-288.

Liu D, Diorio J, Tannenbaum B, Caldji C, Francis D, Freedman A, Sharma S, Pearson D, Plotsky PM, Meaney MJ (1997) Maternal care, hippocampal glucocorticoid receptors, and hypothalamic-pituitary-adrenal responses to stress. Science 277:1659-1662.

Liu D, Diorio J, Day JC, Francis DD, Meaney MJ (2000) Maternal care, hippocampal synaptogenesis and cognitive development in rats. Nat Neurosci 3:799-806.

Maren S (2001) Neurobiology of pavlovian fear conditioning. Annu Rev Neurosci 24:897-931.

Mayford M, Bach ME, Huang YY, Wang L, Hawkins RD, Kandel ER (1996) Control of memory formation through regulated expression of a CaMKII transgene. Science 274:1678-1683.

McEwen BS (2003) Early life influences on life-long patterns of behavior and health. Ment Retard Dev Disabil Res Rev 9:149-154.

Meaney MJ (2001) Maternal care, gene expression, and the transmission of individual differences in stress reactivity across generations. Annu Rev Neurosci 24:1161-1192.

Meaney MJ, Szyf M (2005) Maternal care as a model for experiencedependent chromatin plasticity? Trends Neurosci 28:456-463.

Menard JL, Hakvoort RM (2007) Variations of maternal care alter offspring levels of behavioural defensiveness in adulthood: evidence for a threshold model. Behav Brain Res 176:302-313.

Nemeroff CB (2004a) Early-life adversity, CRF dysregulation, and vulnerability to mood and anxiety disorders. Psychopharmacol Bull 38:14-20.

Nemeroff CB (2004b) Neurobiological consequences of childhood trauma. J Clin Psychiatry 65:18-28. 
Oakley Browne MA, Joyce PR, Wells JE, Bushnell JA, Hornblow AR (1995) Disruptions in childhood parental care as risk factors for major depression in adult women. Aust N Z J Psychiatry 29:437-448.

Ovtscharoff Jr W, Helmeke C, Braun K (2006) Lack of paternal care affects synaptic development in the anterior cingulate cortex. Brain Res 1116:58-63.

Pavlides C, Ogawa S, Kimura A, McEwen BS (1996) Role of adrenal steroid mineralocorticoid and glucocorticoid receptors in long-term potentiation in the CA1 field of hippocampal slices. Brain Res 738:229-235.

Phillips RG, LeDoux JE (1992) Differential contribution of amygdala and hippocampus to cued and contextual fear conditioning. Behav Neurosci 106:274-285

Poeggel G, Helmeke C, Abraham A, Schwabe T, Friedrich P, Braun K (2003) Juvenile emotional experience alters synaptic composition in the rodent cortex, hippocampus, and lateral amygdala. Proc Natl Acad Sci USA 100:16137-16142

Pokorny J, Yamamoto T (1981) Postnatal ontogenesis of hippocampal CA1 area in rats. I. Development of dendritic arborisation in pyramidal neurons. Brain Res Bull 7:113-120.

Pugh CR, Tremblay D, Fleshner M, Rudy JW (1997) A selective role for corticosterone in contextual-fear conditioning. Behav Neurosci 111:503-511.

Sheline YI, Wang PW, Gado MH, Csernansky JG, Vannier MW (1996) Hippocampal atrophy in recurrent major depression. Proc Natl Acad Sci USA 93:3908-3913.

Tang AC, Zou B (2002) Neonatal exposure to novelty enhances long-term potentiation in CA1 of the rat hippocampus. Hippocampus 12:398-404.

Toki S, Morinobu S, Imanaka A, Yamamoto S, Yamawaki S, Honma K (2007) Importance of early lighting conditions in maternal care by dam as well as anxiety and memory later in life of offspring. Eur J Neurosci 25:815-829.
Turner DA, Buhl EH, Hailer NP, Nitsch R (1998) Morphological features of the entorhinal-hippocampal connection. Prog Neurobiol 55:537-562.

van Pelt J, Vajda I, Wolters PS, Corner MA, Ramakers GJ (2005) Dynamics and plasticity in developing neuronal networks in vitro. Prog Brain Res 147:173-188.

Vythilingam M, Heim C, Newport J, Miller AH, Anderson E, Bronen R, Brummer M, Staib L, Vermetten E, Charney DS, Nemeroff CB, Bremner JD (2002) Childhood trauma associated with smaller hippocampal volume in women with major depression. Am J Psychiatry 159:2072-2080.

Weaver IC, Cervoni N, Champagne FA, D’Alessio AC, Sharma S, Seckl JR, Dymov S, Szyf M, Meaney MJ (2004) Epigenetic programming by maternal behavior. Nat Neurosci 7:847-854.

Weaver IC, Champagne FA, Brown SE, Dymov S, Sharma S, Meaney MJ, Szyf M (2005) Reversal of maternal programming of stress responses in adult offspring through methyl supplementation: altering epigenetic marking later in life. J Neurosci 25:11045-11054.

Westbrook RF, Good AJ, Kiernan MJ (1994) Effects of the interval between exposure to a novel environment and the occurrence of shock on the freezing responses of rats. Q J Exp Psychol B 47:427-446.

Whitlock JR, Heynen AJ, Shuler MG, Bear MF (2006) Learning induces long-term potentiation in the hippocampus. Science 313:1093-1097.

Wiegert O, Pu Z, Shor S, Joels M, Krugers H (2005) Glucocorticoid receptor activation selectively hampers $\mathrm{N}$-methyl-D-aspartate receptor dependent hippocampal synaptic plasticity in vitro. Neuroscience 135:403-411.

Wiegert O, Joels M, Krugers H (2006) Timing is essential for rapid effects of corticosterone on synaptic potentiation in the mouse hippocampus. Learn Mem 13:110-113.

Wilson DA, Willner J, Kurz EM, Nadel L (1986) Early handling increases hippocampal long-term potentiation in young rats. Behav Brain Res 21 223-227. 